\title{
Using Real Shadows to Create Virtual Ones
}

\author{
Claus B. Madsen \\ Laboratory of Computer Vision and Image Analysis \\ Aalborg University \\ DK-9220 Aalborg East, Denmark \\ cbm@cvmt.auc.dk \\ http://www. cvmt.auc.dk/ ${ }^{\sim} \mathrm{cbm}$
}

\begin{abstract}
In Augmented Reality (AR) real imagery is superimposed by computer graphics renderings of virtual objects. This paper addresses the problem of creating the illusion that the virtual objects cast credible shadows in the real scenario. A step towards this is to be able to detect shadow regions in images of natural scenes, and this paper describes an approach to such shadow detection. The shadow detection can distinguish between directly lit areas, penumbra (half-shadow) areas, and umbra (full shadow) areas. Furthermore, it is demonstrated how virtual shadows can be created which appear very similar to the real shadows.
\end{abstract}

\section{Introduction}

In Augmented Reality (AR) imagery of real scenes are augmented with visualizations of virtual objects to create the illusion that the virtual objects are a part of the real scene, (see figure 1). AR can be used for, e.g., overlaying disassembly instructions on images of electronic equipment, for interactive entertainment, or for cooperative design and planning, [1,2]. Augmented scenarios can be presented to a user on a computer screen, or via transparent Head Mounted Displays.

Three different issues must be addressed to create truly credible augmentation illusions: 1) registration of virtual coordinate system to scene coordinate system (observer tracking), 2) handling occlusions between real and virtual objects, and 3) subjecting the rendering of virtual objects to virtual lighting which is consistent with the lighting of the real scene.

A typical solution to the registration problem is magnetic tracking technology, or a computer vision based scheme, [3,4]. And the occlusion problem can only be solved properly given a complete $3 \mathrm{D}$ model of the real scene, and then inserting the 3D models of virtual objects in order to solve the visibility issue.

This paper focuses on the latter of the three listed issues, i.e., lighting. The virtual objects must be rendered using a lighting model consistent with the lighting of the real scene. We are especially focusing on being able to cast shadows from virtual objects onto real objects. I.e., creating shadows that are not part of the real scene. In this paper we demonstrate how the image can be classified into areas that are directly lit, areas that are partially lit, and areas that are in shadow, and we demonstrate how these classification results can be used to insert virtual shadows with correct appearances. 

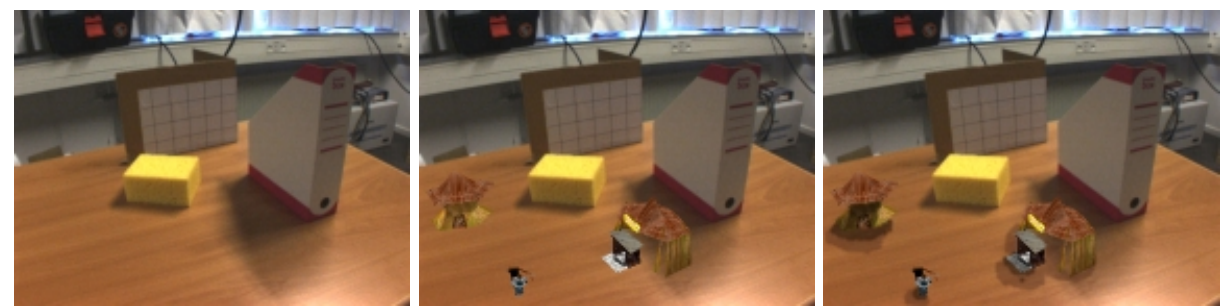

Fig. 1. Illustration of Augmented Reality by superimposing virtual objects on real images, and of how the virtual objects must cast shadows in order to properly appear to be part of the scene. Left: original scene. Middle: virtual objects superimposed on real image. Right: virtual shadows have been manually drawn into the image.

\subsection{Problem Analysis}

There are two main aspects to shadows: 1) the geometric issue, and 2) the spectral issue. The geometric issues deals strictly with the $3 \mathrm{D}$ geometry of the scene and the positions and sizes of light sources. These together determine which areas of the scene will be 1) directly lit, 2) partially lit (penumbra), and 3) in shadow (umbra).

The spectral issue is relevant in cases where there are multiple light sources. For instance outdoor scenes are lit by the sun and the sky (and reflections from objects). This means that areas which are in shadow from the dominant light source(s) are actually lit by the ambient lighting. There have been numerous approaches to estimating lighting conditions in real scenes, either by taking images of reflective spheres placed in the scene, $[5,6]$, or by placing omni-directional cameras in the scene, [7].

Our work is aiming at estimating the scene lighting from the images themselves without imaging the scene with special-purpose objects. This paper addresses a step towards this goal, namely that of detecting real shadows in the images, and using this information to learn how to adjust both the intensity and the color of a region in the image to make it appear as if the region is actually in shadow - a shadow created by a virtual object. We demonstrate in this paper that it is possible to mimic the spectral properties of ambient lighting when creating virtual shadows, as we describe an approach to adjusting pixel colors and intensities so they appear to be in shadow even if they are not in the real image.

The outline of the approach and the paper is as follows. First we perform a color segmentation of the image (section 2 ). Then we automatically compute two thresholds from the intensity histogram of each color segment, thus dividing the pixels into three intensity categories: umbra (lowest intensity), penumbra, and directly lit (highest intensity). Thresholding is described in section 3. Finally, section 4 describes how the information from the real shadows can be used to create virtual shadows using alpha blending. 

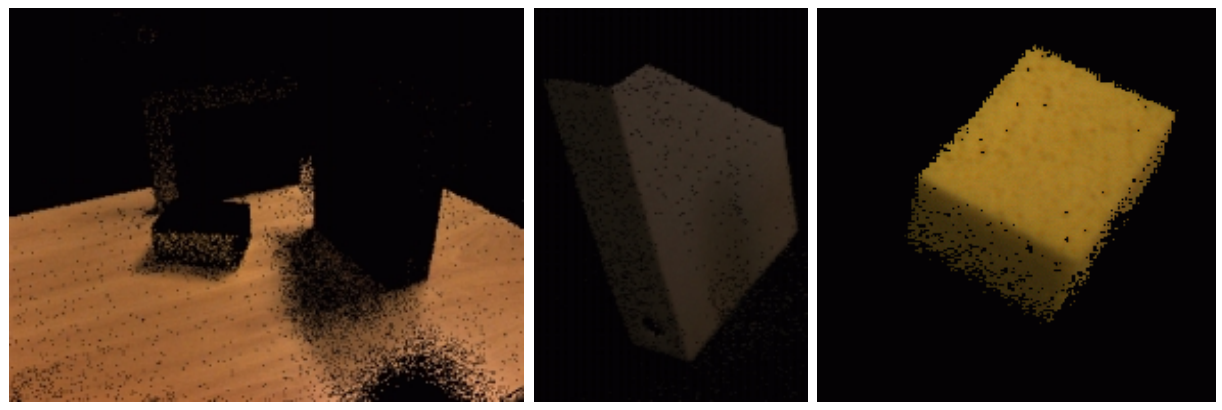

Fig. 2. Left: All pixels of 'table color' segmented from the image in figure 1. Middle and right: paper holder and sponge segmented from another image (now shown).

\section{Segmentation of Regions}

Figure 2 illustrate how it is possible to segment all pixels of similar color. The approach taken is to convert the RGB color image to the HSV color space. Then we form classes in the $(\mathrm{H}, \mathrm{S})$ plane by computing the mean and the covariance matrix for a small population of pixels with similar color. For the examples given in this paper the training is interactive in the sense that the user selects a small rectangular area, for example on the table in figure 1. The mean for this class, $\mu_{c}=\left[\mu_{H}, \mu_{S}\right]$, and the 2 by 2 covariance matrix, $\Sigma_{c}$, is computed. Subsequently the squared Mahalanobis distance, $D^{2}\left(p_{i}, c\right)$, from any given pixel, $p_{i}=\left[H_{i}, S_{i}\right]$, to the class $c$ is computed:

$$
D^{2}\left(p_{i}, c\right)=\left[\mu_{c}-p_{i}\right] \cdot \Sigma_{c} \cdot\left[\mu_{c}-p_{i}\right]^{T}
$$

If the squared Mahalanobis distance, $D^{2}\left(p_{i}, c\right)$, is below a threshold, the pixel $p_{i}$ is classified as belonging to the color class $c$. In the present implementation this threshold has been set corresponding to a confidence level of $99 \%$.

\section{Classification of Shadows}

The intensities naturally hold a lot of information about how much light hits a region in the scene. Figure 3 shows the intensity histogram for the table region shown in figure 2. In [8] a somewhat heuristic approach is taken to intensity threshold selection based on detecting histogram peaks by differentiation, which is a fragile approach. We demonstrate here that the thresholds can be selected completely automatically using a statistically based scheme.

Assume that the intensity histogram has been normalized with the number of pixels so as to represent a probability density function for intensity values. Intensity values lie in the range $\left.] 0 ; I_{\max }\right]$. Thus, the histogram value at intensity $i, P(i)$, gives the probability of this intensity value in the image. The sum of $P(i)$ over all $i$ equals 1 . 


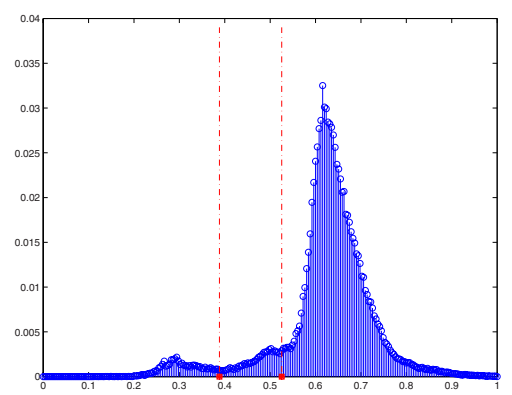

Fig. 3. Intensity histogram for all pixels with table color from figure 2. The two (red) vertical lines on the horizontal axis indicate two threshold values found automatically, separating the pixels into 3 categories: umbra (below lowest threshold), penumbra, and directly lit (above highest threshold).

An approach exists, developed by Otsu, $[9,10]$, for automatically selecting a single threshold given such a histogram (assumed to be bi-modal), The approach finds the threshold which minimizes the within-group variance. For a given threshold, $t$, the within-group variance is found as:

$$
\begin{gathered}
\sigma_{w}^{2}(t)=q_{1}(t) \sigma_{1}^{2}(t)+q_{2}(t) \sigma_{2}^{2}(t) \\
q_{1}(t)=\sum_{i=1}^{t} P(i) \quad \text { and } \quad q_{2}(t)=\sum_{i=t}^{I_{\max }} P(i)
\end{gathered}
$$

and where $\sigma_{1}^{2}(t)$ is the group variance of the population below the threshold $t$, and $\sigma_{2}^{2}(t)$ is the group variance of the population above. The within-group variance is computed using eq. 2 for all possible threshold values, $\left.t \in] 0 ; I_{\max }\right]$. The threshold value which yields the smallest within-group variance is then selected. This threshold selection scheme has been implemented and used in the following manner:

1. use the threshold selection approach to find a first threshold, $t_{1}$, separating the population into two: directly lit, and not directly lit

2. remove all pixels with intensity above $t_{1}$ from the population, i.e., remove the directly lit pixels

3. compute new intensity histogram for the remaining pixel population (the non-directly lit)

4. automatically select a second threshold, $t_{2}$, separating non-directly lit pixels into umbra and penumbra

This two step approach was used to generate the lighting classification shown in figure 4 . The approach works very well if the pixel population is dominated by directly lit pixels, i.e., if there is much more directly lit area than umbra and/or penumbra. This is not the case for the paper holder of figure 2. Figure 5 shows the histogram for this pixel population. 

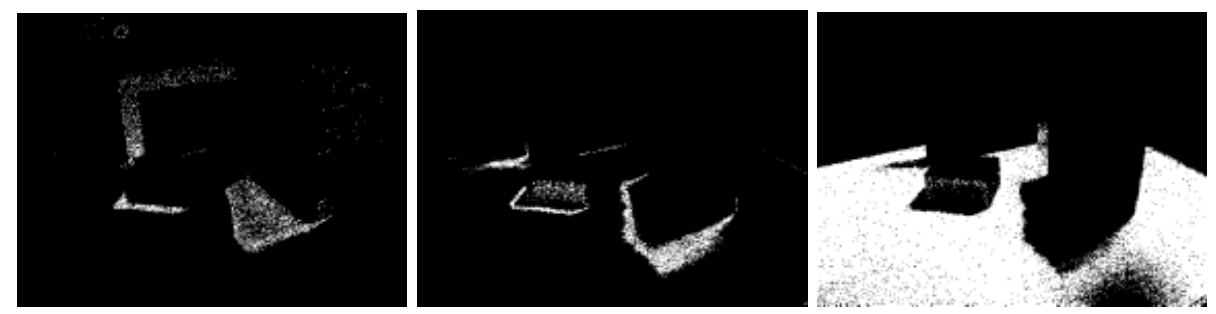

Fig. 4. Left: pixels of the table surface classified as being lit only by ambient lighting (umbra). Middle: pixels partially lit by the light source, (penumbra). Right: pixels directly lit by the dominant light source.
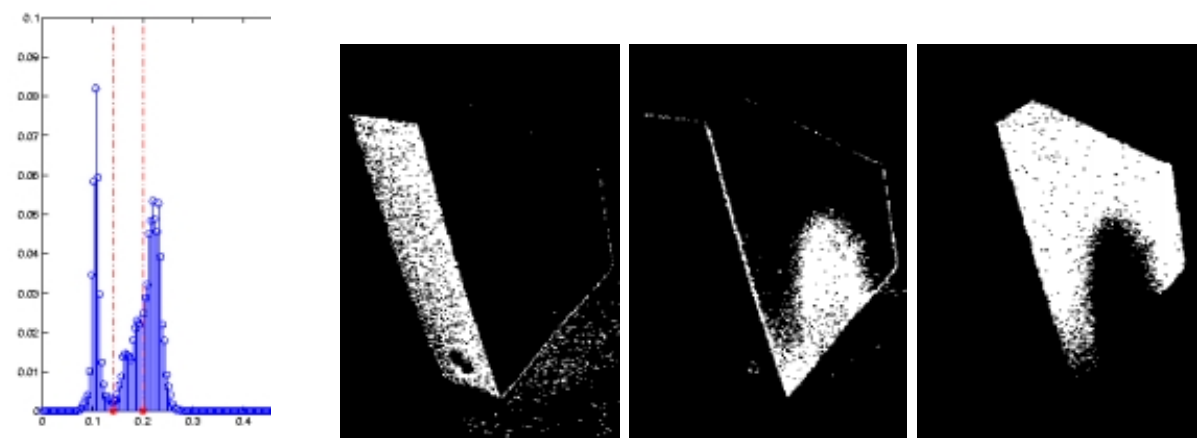

Fig. 5. Far left: Intensity histogram for all pixels of the paper holder from figure 2 . The two (red) vertical lines indicate the two thresholds determined simultaneously. Last three images: umbra area, penumbra area, and directly lit area, respectively.

To address this problem we have generalized the threshold selection scheme from $[9,10]$ to tri-modal histograms, enabling the method to simultaneously find the combination of two thresholds, $t_{1}$, and $t_{2},\left(t 2>t_{1}\right)$, minimizing the withingroup variance:

$$
\begin{gathered}
\sigma_{w}^{2}\left(t_{1}, t_{2}\right)=q_{1}\left(t_{1}\right) \sigma_{1}^{2}\left(t_{1}\right)+q_{2}\left(t_{1}, t_{2}\right) \sigma_{2}^{2}\left(t_{1}, t_{2}\right)+q_{3}\left(t_{2}\right) \sigma_{3}^{2}\left(t_{2}\right) \\
q_{1}(t)=\sum_{i=1}^{t} P(i) \quad, \quad q_{2}\left(t_{1}, t_{2}\right)=\sum_{i=t_{1}}^{t_{2}} P(i), \text { and } \quad q_{3}\left(t_{2}\right)=\sum_{i=t_{2}}^{I_{\text {max }}} P(i)
\end{gathered}
$$

Figure 5 shows the thresholds chosen by simultaneous selection of both thresholds, which in this case performs much better than the two-step approach described above. The light classification results are also shown.

\section{Generating Fake Shadows}

We now turn towards attempting to create virtual shadows, which mimic real shadows. We will not address how to compute where to place virtual shadows, 
but only look at how to change the spectral properties of an image area so that it looks like a real shadow. Techniques for computing where to cast shadows can be found in $[11,12]$.

The section presents a scheme enabling us to change the spectral properties of one pixel population, so that it equals the properties of some other population. That way we can for example take a directly lit area and turn it into what it would look like if it had actually been a penumbra area. Figure 6 illustrates the result.
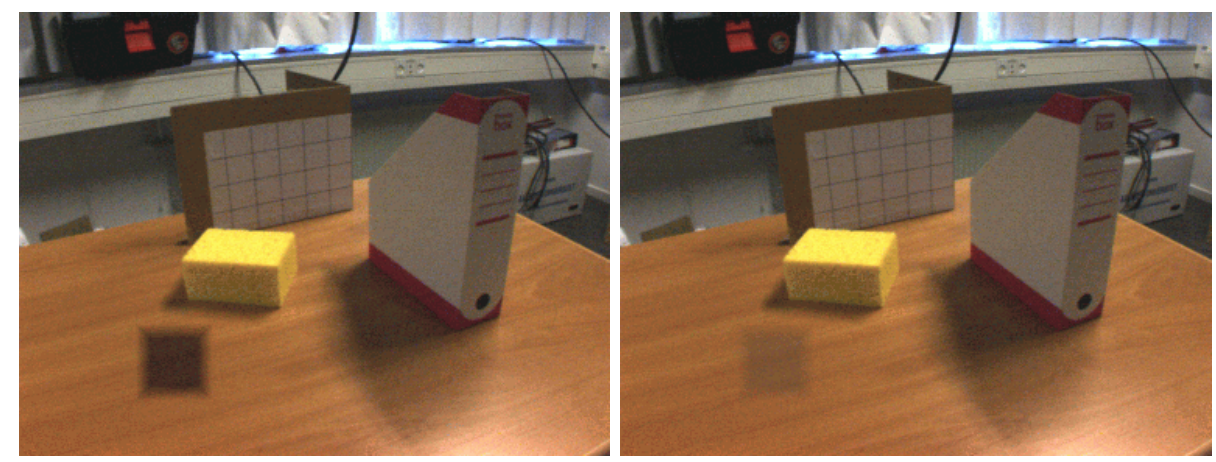

Fig. 6. A fake umbra area (left) and penumbra area (right) has been added to the image to illustrate that image regions can be adjusted in terms of intensity and color so as to appear as shadow.

Assume we have an image region from the original image with some average RGB values, $[\bar{R} \bar{G} \bar{B}]_{o}^{T}$. This region can for example be a directly lit region in the image. Assume also that we have a 'model' region, with average RGB values $[\bar{R} \bar{G} \bar{B}]_{m}^{T}$, for example an umbra region in the same image. We wish to change the original so that it has the same color and intensity as the model.

We will use the image blending scheme known as alpha blending, [12], to change the appearance of the original region, so that it acquires the same average as the model region. Alpha blending is performed on all pixels in a defined area and is supported by contemporary computer graphics hardware. In its general form alpha blending for a pixel $i$ is a linear combination of the pixel's original RGB values and an alpha overlay:

$$
\left[\begin{array}{c}
R_{i} \\
G_{i} \\
B_{i}
\end{array}\right]_{r}=\alpha\left[\begin{array}{c}
R_{i} \\
G_{i} \\
B_{i}
\end{array}\right]_{a}+(1-\alpha)\left[\begin{array}{c}
R_{i} \\
G_{i} \\
B_{i}
\end{array}\right]_{o}
$$

where subscript $r$ indicates the result RGB values after the alpha blending, subscript $a$ indicates alpha overlay RGB values, and subscript $o$ indicates the pixel's original RGB values before blending.

Eq. 6 means that we can change for example a directly lit region into an umbra region, provided we can find a combination of an $\alpha$ value and an overlay 
RGB vector, $[R G B]_{a}^{T}$, which when applied to all pixels in a region changes the average from $[\bar{R} \bar{G} \bar{B}]_{o}^{T}$ to $[\bar{R} \bar{G} \bar{B}]_{m}^{T}$. I.e., we need to solve the following equation system for $\alpha, R_{a}, G_{a}$, and $B_{a}$ :

$$
\left[\begin{array}{c}
\bar{R} \\
\bar{G} \\
\bar{B}
\end{array}\right]_{m}=\alpha\left[\begin{array}{l}
R \\
G \\
B
\end{array}\right]_{a}+(1-\alpha)\left[\begin{array}{c}
\bar{R} \\
\bar{G} \\
\bar{B}
\end{array}\right]_{o}
$$

We will use the same RGB values for all pixels in the alpha overlay, therefore there is no subscript $i$ and no average bar over the overlay's RGB components in eq. 7 . If we briefly treat $\alpha$ as a known value we can re-arrange eq. 7:

$$
\begin{aligned}
R_{a} & =\frac{1}{\alpha}\left(\bar{R}_{m}-\bar{R}_{o}\right)+\bar{R}_{o} \\
G_{a} & =\frac{1}{\alpha}\left(\bar{G}_{m}-\bar{G}_{o}\right)+\bar{G}_{o} \\
B_{a} & =\frac{1}{\alpha}\left(\bar{B}_{m}-\bar{B}_{o}\right)+\bar{B}_{o}
\end{aligned}
$$

In principle we could choose any value for $\alpha$, and there would still be a solution to eqs. 8 through 10 . But, there are other constraints to consider. First of all $\alpha$ must be in the interval $[0 ; 1]$. Secondly, $\alpha$ should be as small as possible in order for the blending represented by eq. 6 to preserve as much as possible of the original pixel values $\left(\left[R_{i} G_{i} B_{i}\right]^{T}\right)$, i.e., to weigh the original values as high as possible. This will ensure that the original texture will be visible through the alpha shadow overlay. On the other hand, hardware supported alpha blending does not allow any of the components $\left[R_{i} G_{i} B_{i}\right]^{T}$ in the alpha overlay to be negative. This last constraint translates into three constraints on how small $\alpha$ can be, one for each color component, where $\alpha$ then has to be higher than or equal to the largest of the three values:

$$
\alpha \geq \max \left(\frac{\bar{R}_{o}-\bar{R}_{m}}{\bar{R}_{o}}, \frac{\bar{G}_{o}-\bar{G}_{m}}{\bar{G}_{o}}, \frac{\bar{B}_{o}-\bar{B}_{m}}{\bar{B}_{o}}\right)
$$

Since $\alpha$ has to be small to preserve texture, we choose the smallest value satisfying eq. 11. The steps in creating a virtual shadow then become:

1. take a small population of pixels which are in shadow, e.g., penumbra, and compute the average RGB values, $[\bar{R} \bar{G} \bar{B}]_{m}^{T}$

2. take the population of pixels in the region which should be changed into appearing like shadow and compute the average RGB values, $[\bar{R} \bar{G} \bar{B}]_{o}^{T}$

3. apply eq. 11 to find the best $\alpha$ value

4. apply eqs. 8 through 10 to compute the RGB components of the alpha overlay, $[R G B]_{a}^{T}$

5. perform alpha blending using eq. 6 using the same RGB values for all pixels in the overlay 


\section{Conclusions and Future Work}

It has been demonstrated that images of natural scenes can be classified into directly lit, penumbra or umbra regions without any prior knowledge of lighting or scene content. It has also been demonstrated that given this classification it is possible to add realistic false shadows to images, showing that it is possible to let virtual objects cast shadows in real scenes.

Future research involves developing methods for estimating light source positions from images, and using this information to generate renderings of virtual objects, including shadows, and superimposing them on images.

\section{Acknowledgments}

This research is funded in part by the ARTHUR project under the European Commission IST program (IST-2000-28559), and in part by the BENOGO project (IST-2001-39184). This support is gratefully acknowledged.

\section{References}

1. Azuma, R.T.: A survey of augmented reality. Presence: Teleoperators and Virtual Environments 6 (1997) $355-385$

2. Azuma, R.T., Baillot, Y., Behringer, R., Feiner, S., Julier, S., MacIntyre, B.: Recent advances in augmented reality. IEEE Transactions on Computer Graphics and Applications 21 (2001) $34-47$

3. State, A., Hirota, G., Chen, D.T., Garrett, W.F., Livingston, M.A.: Superior augmented-reality registration by integrating landmark tracking and magnetic tracking. In: Proceedings: Conference on Computer Graphics and Interactive Techniques (SIGGRAPH'96), New Orleans, Louisiana. (1996) 429 - 438

4. Trucco, E., Verri, A.: Introductory Techniques for 3D Computer Vision. Prentice Hall (1998)

5. Powell, M.W., Sarkar, S., Goldgof, D.: A simple strategy for calibrating the geometry of light sources. IEEE Transactions on Pattern Analysis and Machine Intelligence 23 (2001) $1022-1027$

6. Debevec, P.: Image-based lighting. IEEE Transactions on Computer Graphics and Applications (2002) $26-33$

7. Sato, I., Sato, Y., Ikeuchi, K.: Acquiring a radiance distribution to superimpose virtual objects onto a real scene. IEEE Transactions on Visualization and Computer Graphics 5 (1999) 1 - 10

8. Funka-Lea, G.D.: The Visual Recognition of Shadows by an Active Observer. PhD thesis, University of Pennsylvania (1994)

9. Otsu, N.: A threshold selection method from gray-level histograms. IEEE Transactions on Systems, Man, and Cybernetics SMC-9 (1979) 62 - 66

10. Haralick, R.M., Shapiro, L.G.: Computer and Robot Vision, Vol. 1. AddisonWesley (1992)

11. Foley, J.D., van Dam, A., Feiner, S.K., Hughes, J.F., Phillips, R.L.: Introduction to Computer Graphics. Addison-Wesley (1994)

12. Watt, A., Policarpo, F.: 3D Games: Real-Time Rendering and Software Technology. Volume 1. Addison-Wesley (2001) 